\title{
A Brief Introduction of Paradox in Physics
}

\author{
Narayan Prasd Dahal
}

\author{
P.N. Campus, Pokhara, email: the_npd1@yahoo.com
}

A paradox is a situation which gives one answer when analyzed in one way and a different answer when analyzed in another way, so that we are left in somewhat from actually what should happen. Of course, in physics there are never any paradoxes because there is only one correct answer that we believe as nature will act in only one way and that is the right way naturally. The present apparent paradox has important applications, especially in atomic physics and therefore has been verified experimentally. Some of the paradox or confusion in this nature is resolved from the expert of the same field but some are still as the question mark for them. So the physicists define the paradox as the only confusion in their own understanding to the relevant matter. There are so many paradoxes in physics, out of them most important six paradoxes are mentioned below:

\section{The Barn and ladder paradox:}

The paradox of Barn and ladder is originated from the concept of Lorentz contraction. As there is no direct experimental conformation of Lorentz contraction simply because it is too difficult to get an object of measurable size going any where near the speed of light. In the way to Barn and ladder paradox, once there was a farmer who had a ladder which was too long to store in his barn. One day he got chance to read some relativity and a solution to his problem suggested itself. He instructed his daughter to run with the ladder as fast as she could - the moving ladder having Lorentz contracted to a size that the barn could easily accommodate, she was to rush through the door where upon the farmer would slam it behind her capturing the ladder inside the barn. The daughter of farmer however has read somewhat more in the relativity book, she points out that in her reference frame the barn, not the ladder will contract, and the fit will be even worse that it was with the two at rest .
Who is right? Will the ladder fit inside the barn, or won't it? This becomes a paradox and is named as barn and ladder paradox.

Solution is they are both right! When you say "the ladder is in the barn" you mean that all parts of it are inside at one instant of time, but in view of the relativity of simultaneity, that is a condition that depends on the observer. There are two relevant events here:

a) Back end of ladder makes it in the door.

b) Front end of ladder hits far wall of barn

The farmer says (a) occurs before (b) so there is a time when the ladder is entirely within the barn, his daughter says (b) precedes (a), so there is not ladder within the barn.

\section{Ehrenfest's paradox:}

This paradox is first analyzed by Paul Ehrenfest in 1909 and it is related to the relativistic geometry, also it is concerned to the rotation of a rigid disc in the theory of relativity. It discusses an ideally rigid cylinder that is made to rotate about its axis of symmetry. The radius $\mathrm{R}$ as seen in the laboratory frame is always perpendicular to its motion and should therefore be equal to its value Ro when stationary.

To illustrate this paradox let us consider a record turn table of radius $\mathrm{R}$ is rotated with an angular velocity $\mathrm{w}$. The circumference of the turntable is assumed to be Lorentz-contracted but the radius of the turntable is not contracted as by Lorentz because it is perpendicular to the velocity. If we wanted to find out the ratio of circumference to the diameter then from the rules of ordinary geometry, the ratio must be pie. Now the contradictory question, are we finding the ratio of circumference and diameter to be pie? What is the result? There exists a paradox and is called Ehrenfest's paradox. 


\section{Merzbacher paradox:}

The paradox is captured from the electromagnetism by Somerfield. It tells, let us consider a long straight wire carrying current I having surface area ' $\mathrm{s}$ ' then from the Biot-Savart law, magnetic field outside a long straight wire carrying steady current is I

$$
B=\mu_{0} \frac{I}{2 \pi s}
$$

Also the electric field inside the wire is uniform i.e.

$$
E=\frac{I \rho}{\pi a^{2}}
$$

Where $\rho$ is the resistivity, ' $a$ ' is the radius of wire. Now, the intersecting question is that what is the value of electric field outside the wire? This is the famous problem first analyzed by Sommerfield and followed by Merzbacher so it is incarnation recently as Mertzbacher paradox.

\section{Gibb's paradox}

Gibb's paradox, from the name it is clear that the paradox is first known by Josian Williard Gibbs in 1992 and at the same time he developed formulation of statistical mechanics in terms of concept of ensembles. The name Canonical ensemble is also originated by Gibbs when he was at Yale University.

To analyze the Gibb's paradox, consider two ideal gases having no. of particles $\mathrm{N}_{1}$ and $\mathrm{N}_{2}$ kept in two separate volumes $\mathrm{V}_{1}$ and $\mathrm{V}_{2}$ at the same temperature at the same density. When the gases are allowed to mix then the change in entropy becomes;

$$
S=N_{1} K \cdot \log \frac{V}{V_{1}}+N_{2} K \cdot \log \frac{V}{V_{2}}
$$

This is positive quantity. For the expression, $\mathrm{V}=\mathrm{V}_{1}+\mathrm{V}_{2}$.

If the gases are different then the result is experimentally correct. Now, if we consider the mixing of same kind of gas then paradox present it. Since the change in entropy does not depend on the identity of gases and thus cannot be a function of thermodynamic state alone. As the entropy of mixing for the same kind of gases should be zero but we found it to be positive quantity.
What is the entropy of mixing of same kind of gas, positive quantity or zero? Gibbs resolved the paradox in an empirical fashion by postulating that there is an error in calculating no. of states of all the identical molecules and hence Gibbs assumed that the correct answer is $\mathrm{N}$ ! times smaller than it thought was, in the calculation of no. of states of identical particles.

\section{Feynmann disk paradox:}

The paradox is taken out from the Feynman lectures delivered in 1963. It is originated from electro dynamical mechanics. To illustrate the paradox let us consider a device consisting of thin, circular plastic disk supported on concentric bearings so that it is quite free to rotate. On the disk there is a coil of wire in the form of a short solenoid concentric with the axis of rotation. This solenoid carries a steady current I provided by battery. Near the edge of the disc around its circumference there are no. of small metal spheres insulated from each other and also from solenoid by plastic material are spaced uniformly. Each of them is charged with the same electrostatic charge. Suppose if the current is continued then there was a magnetic flux through the solenoid more or less parallel to the axis of disc. Now, if the current is interrupted, this flux must go to zero. Then there will be an electric field induced. Then the charge spheres will be all experience an electric field and electric force in all charges provides a net torque on the disc. From these arguments we would expect that as the current in the solenoid disappear disc would begin to rotate. If we knew the moment of inertia of the disc, the current in the solenoid and the charges of small spheres, we can find the angular velocity.

But there could be also a different argument using the principle of conservation of angular momentum of the disc with all its equipment is initially zero and so the angular momentum of the assembly should remain zero. There should be no rotation when the current is stopped. Which of the above arguments is correct? Will the disc rotate or will not rotate when the current is stopped? This is the famous paradox lectured by Feynmann and also known as Fenymann disk paradox. 


\section{Twins paradox:}

The twin's paradox is totally related to the relativity and it is proposed by Albert Einstein in 1911. Einstein original statement of the twin paradox is "if we placed a living organism in a box...one could arrange that the organism, after an arbitrary lengthy flight could be returned to its original spot in a scarcely altered condition, while corresponding organisms which had remained in their original positions had long since given way to new generations."

Consider a particle moving in the laboratory system with a velocity $v$ that is not constant. Since the system in which the particle is at rest is accelerated with respect to the laboratory system then the two systems should not be connected by a Lorentz transformation. It is assumed that this Lorentz transformation will also derived the properties of the particles. From Lorentz inverse transformation,

$$
t=\frac{t_{0}}{\sqrt{1-\frac{V^{2}}{c^{2}}}},
$$

where to is the time measured by a clock at rest and $t$ is the measured at velocity $\mathrm{v}$.

Now, to show twins paradox considers two exactly identical twins brothers. Let one of them go to the long space journey at a high speed in a rocket and the other stay behind on the earth. The clock in the moving rocket will appear to go slower than the clock on the earth in accordance with $t=t_{0} /\left(1-v^{2} /\right.$ $\left.c^{2}\right)^{1 / 2}$. Therefore when he returns back to the earth, he will find himself younger than the twin who stayed behind on the earth.

Can twins be elder and younger? This is the interesting paradox in carded as twin's paradox. From the resolution of twin's paradox it is concluded that "moving clock run slow." 